\title{
Development and validation of chromatographic methods for screening and subsequent quantification of suspected illegal antimicrobial drugs encountered on the Belgian market
}

\author{
Yaxin Tie $^{\mathrm{a}, \mathrm{b}}$, Celine Vanhee ${ }^{\mathrm{a}}$, Eric Deconinck ${ }^{\mathrm{a}, 1}$, Erwin Adams $^{\mathrm{b}, 1, *}$ \\ ${ }^{a}$ Scientific Direction Chemical and Physical Health Risks, Section Medicines and Healthcare Products, Sciensano, J. Wytsmansstraat 14, 1050 Brussels, Belgium \\ ${ }^{\mathrm{b}}$ KU Leuven, University of Leuven, Department of Pharmaceutical and Pharmacological Sciences, Pharmaceutical Analysis, Herestraat 49, O\&N2, PB 923, B-3000 Leuven, \\ Belgium
}

\section{A R T I C L E I N F O}

\section{Keywords:}

UHPLC-DAD

UHPLC-MS ${ }^{2}$

Substandard and falsified antimicrobials

Accuracy profiles

\begin{abstract}
A B S T R A C T
Estimations, made by the World Health Organization (WHO), state that $10 \%$ of the medical products in low- and middle-income countries are substandard or falsified (SF). Among them, antibiotics and antimalarials are the most commonly reported since 2013. Besides the fact that falsification is a crime, the worldwide use of poor quality antimicrobials could result in treatment failures, stronger antimicrobial resistance and even the promotion of the emergence of superbugs. Therefore, simple and accurate analytical methods are necessary, which are capable to detect and quantify a wide range of antimicrobials in suspected illegal products. In this work, a screening and a quantification method using ultra-high performance liquid chromatography with tandem mass spectrometry (UHPLC-MS ${ }^{2}$ ) and diode array detection (UHPLC-DAD), respectively were developed and validated. These methods could be used for routine analysis and enable a more in-depth characterization of SF-antimicrobials.

According to their popularity as SF-antimicrobials, 31 antibiotics, 3 antibacterial agents, 1 antifungal agent and 1 beta-lactamase inhibitor, covering eleven different antibacterial classes, were selected. The UHPLC-MS ${ }^{2}$ screening method with gradient elution is able to selectively detect these 36 compounds within 18 min (including wash and equilibration step). It was validated for sensitivity, selectivity and matrix effects. Within an analysis time of $32 \mathrm{~min}$, the UHPLC-DAD method could quantify 32 compounds (4 showed insufficient UV absorbance) and resulted in sufficient selectivity, necessary since some SF-antimicrobials may include more than one antimicrobial component. This quantification method was validated for the positive hits found during screening tests of suspected illegal samples. This resulted in a validation set of 11 antimicrobials and 1 betalactamase inhibitor. The "total error" approach in accordance with the validation requirements of ISO-17025 was employed for the validation. 57 real-life illegal samples, seized by inspectors from the Belgium Federal Agency for Medicinal and Health Products (FAMHP), were analyzed using the two described methods. About half of them were not compliant and some samples that contained clavulanic acid showed a serious reduction in the amount of this molecule (in one sample only $14 \%$ of the claimed dosage was found). These quality issues might be attributed to either poor manufacturing, storage or transportation conditions.
\end{abstract}

\section{Introduction}

Despite the multitude of local and global efforts that were made during the last decade to curtail the manufacturing, distribution and supply of substandard and falsified (SF) medicines, these products are still considered a continuously growing threat to public health [1]. Estimations made by the World Health Organization (WHO) state that $10 \%$ of the medical products in low- and middle-income countries are
SF. Among them, antibiotics and antimalarials are the most commonly reported since 2013 [2]. Moreover, it seems that antibiotics account for more than $25 \%$ of all SF-drugs [3]. This is likely due to the success of the antibiotic market since it has been reported that this had a revenue of USD 39.8 billion in 2015 and is even expected to grow up to USD 57.0 billion by 2024 [4]. Recent estimates claimed that the illegal antibiotic market occupied around 5\% of the global antibiotic market [3]. In addition, a warning issued by WHO stated that SF-antibiotics are no

\footnotetext{
* Corresponding author.

E-mail address: Erwin.Adams@kuleuven.be (E. Adams).

${ }^{1}$ Equally contributing project leaders.
} 
longer only limited to developing countries, but are also gaining popularity in the developed ones. This phenomenon is attributed to the expansion of the internet and the ease of online purchasing. Although it is well known that more than $50 \%$ of the medicines sold by web shops not disclosing their physical identity are SF-medicines, a recent survey conducted in the United States indicated that the number of people purchasing drugs online has increased by three times in the past decade [2]. Moreover, it was reported that in total about 21,000 dose units of SF-antibiotics were seized in 12 European countries within one week in 2012 [5].

According to $\mathrm{WHO}, \mathrm{SF}$ medical products may include products exclusively containing inactive ingredients, wrong ingredients or improper dosages [6]. SF-antimicrobials with insufficient dosages may result in sub-potent drug efficacy, leading to increased morbidity of patients, treatment failures and selection of drug-resistant microbial strains. SF-antimicrobials with excessive dosages or impurities, can also directly be responsible for serious side effects and even deathly outcomes, especially in children [7]. A case report, originating from the late 1990s showed that the use of SF-gentamicin, containing toxic impurities, caused around 2000 adverse incidents of the eosinophiliamyalgia syndrome and resulted also in 66 deaths in the United States [3]. The use of SF-antimicrobials worldwide may also result in stronger antimicrobial resistance and even promotes the emergence of superbugs [8]. Therefore, great efforts are currently ongoing to tackle these malignant practices in favor of public health. In this framework, not only regulatory efforts are required, but also adequate analytical methods are paramount. In literature, many validated methodologies are available for the identification and quantification of antimicrobials, but most of them are designed for one specific class of antimicrobials or are analyzed by a targeted approach [9-17]. These approaches are known to be selective and sensitive, but have an inherent bias since they will not detect molecules other than the preselected ones. Therefore, a full screening method is necessary in order to detect unexpected SF-antimicrobial drug products and possible adulterants. Moreover, the European pharmacopoeia methods for antibiotics are designed only for one specific antibiotic [18] which require different reagents and different experimental conditions. Therefore, these methods are not feasible for high throughput screening of SF-antimicrobials. Potential adulterants in falsified samples are not able to be properly detected using the European pharmacopoeia methods. Given the possible high amount of suspected antibiotics seized at a certain moment, a fast and selective analytical technique is demanded by regulatory authorities to monitor drug quality control and survey the (illegal) market.

This study aims to develop such a method capable of efficient detection and sufficient separation of suspected illegal antimicrobials frequently encountered by the Belgian controlling agency. Besides real antibiotics, the set included also a beta-lactamase inhibitor (clavulanic acid), an antifungal (griseofulvin) and some antibacterial agents (trimethoprim, sulfamethoxazole and nitrofurantoin). The best performing LC gradient was selected, further refined and validated for identification by mass spectrometry (MS) and quantification by a diode array detector (DAD). For quantification, DAD was preferred over MS because the first provides more accurate results without the need of expensive isotopically labelled reference standards. Good accuracy was required in view of acceptance limits of $\pm 5 \%$ for the assessment of the actives in pharmaceutical products (tablets and capsules). All collected illegal antimicrobial samples were successfully screened and quantified using the validated methods.

\section{Materials and methods}

\subsection{Reagents and standards}

\subsubsection{Reagents}

HPLC and MS-grade acetonitrile (ACN) and methanol were obtained from Biosolve (Valkenswaard, the Netherlands). Formic acid (ACS and
Ph. Eur. reagent) was purchased from Merck (Darmstadt, Germany). Water $(\mathrm{R}=18 \mathrm{M} \Omega \mathrm{cm}$, TOC $<4 \mathrm{ppb}$ ) was generated by using a milliQGradient A10 system (Millipore, Billerica, MA, USA).

\subsubsection{Standards and stock solutions}

The reference standards of amoxicillin trihydrate, azithromycin, cefaclor, ceftazidime, cefazolin, cefuroxime axetil, clavulanate lithium, clindamycin and cloxacillin sodium were purchased from the European Directorate for the Quality of Medicines (EDQM) (Strasbourg, France). Ampicillin, cefadroxil, cefotaxime sodium, cefradine, ceftriaxone disodium salt heptahydrate, erythromycin A, tetracycline hydrochloride and roxithromycin were obtained from Sigma-Aldrich (St. Louis, MO, USA). Benzathine penicillin G tetrahydrate, cefalexin, doxycycline hyclate, lincomycin hydrochloride monohydrate, penicillin $\mathrm{V}$ potassium, trimethoprim and norfloxacin were bought from Sigma-Aldrich (Seelze, Germany), while ciprofloxacin and rifampicin were acquired from Sigma-Aldrich (Buchs, Switzerland). Bacitracin zinc was purchased from Alpharma (Oslo, Norway). Clarithromycin and ofloxacin were obtained from Ranbaxy (Madhya Pradesh, India). Nitrofurantoin, sulfamethoxazole and neomycin sulfate were obtained from Fagron (Waregem, Belgium). Griseofulvin was purchased from Sigma-Aldrich (Laramie, WY, USA), polymyxin B sulfate from Kela laboratoria N.V. (Hoogstraten, Belgium) and cefepime from Bristol-Myers Squibb (Syracuse, New York, USA). Gentamicin sulfate was obtained from Alcon Cusi (Barcelona, Spain).

For the sample matrix components, magnesium trisilicate was purchased from Qualiphar (Bornem, Belgium). Starch was obtained from VWR (Darmstadt, Germany) and lactose monohydrate was bought from Synergyhealth (Uitgeest, Netherlands). Mannitol, croscarmellose sodium and cellulose microcristalline were obtained from Fagron (Waregem, Belgium). Sucrose, propylparaben and methylparaben were from Sigma-Aldrich (St. Louis, MO, USA). Maltodextrin was bought from Procter \& Gamble (Strombeek-Bever, Belgium).

Individual stock solutions were prepared by dissolving standards in the corresponding solvent (See Table 1 ) at a concentration of $1 \mathrm{mg} / \mathrm{mL}$. Afterwards, stock solutions were divided in different aliquots and stored at $-20^{\circ} \mathrm{C}$ for maximum six months whilst quinolones were refreshed every two months and $\beta$-lactams renewed monthly, as recommended by Gros et al. [11]. It is noted that tetracyclines have to be stored in the dark due to photodegradation [19]. The standard mixture solution containing all antimicrobials was prepared in a mixture of methanol $/ \mathrm{H}_{2} \mathrm{O}(50: 50, \mathrm{v} / \mathrm{v})$ by mixing proper amounts of individual stock solutions at a concentration of $25 \mu \mathrm{g} / \mathrm{mL}$. The standard mixture solution was stored at $-20{ }^{\circ} \mathrm{C}$ and renewed every week.

\subsection{Samples of suspected illegal antimicrobials}

All illegal samples were seized and collected by inspectors from the Belgium Federal Agency for Medicines and Health Products (FAMHP) during the period 2016-2017. 57 samples were included in the study, claiming 18 different antimicrobials and 1 beta-lactamase inhibitor: amoxicillin, ampicillin, azithromycin, benzathine penicillin G, ceftriaxone, cefalexin, ciprofloxacin, doxycycline, erythromycin, griseofulvin, ofloxacin, penicillin $\mathrm{V}$, roxithromycin, tetracycline (hydrochloride), nitrofurantoin, norfloxacin, sulfamethoxazole, trimethoprim and clavulanic acid. This set was further completed with 17 other antimicrobials, based on their popularity in literature as SF-antimicrobials $[3,5,7]$. Taken together, a total 36 compounds were included for method development, covering eleven different classes of antimicrobials (See Table 1). The collected 57 illegal samples consisted of four different formulations: capsules (46\%), tablets (47\%), injections $(5 \%)$ and syrup (2\%). It is demonstrated that counterfeit $\beta$-lactams are frequently encountered by the controlling agency according to existing criminal cases $[3,7]$. Conformably, two thirds of the collected counterfeit products in the present study are $\beta$-lactam antibiotics. 
Table 1

List of compounds for the UHPLC-MS ${ }^{2}$ screening method and the UHPLC-DAD quantification method. Solvent A is methanol/ $\mathrm{H}_{2} \mathrm{O}$ mixture $(50: 50, \mathrm{v} / \mathrm{v})$, solvent $B$ is methanol and solvent $\mathrm{C}$ is methanol/ $\mathrm{H}_{2} \mathrm{O}$ with $1 \% \mathrm{HCOOH}$ mixture $(20: 80, \mathrm{v} / \mathrm{v})$.

\begin{tabular}{|c|c|c|c|}
\hline Compounds & Classification & Solvent & $\begin{array}{l}\text { Wavelength } \\
\text { (nm) }\end{array}$ \\
\hline Amoxicillin & Penicillins & A & 230 \\
\hline Ampicillin & Penicillins & A & 230 \\
\hline Azithromycin ${ }^{1}$ & Macrolides & $\mathrm{B}$ & \\
\hline Bacitracin $\mathrm{Zn}$ & Polypeptides & $\mathrm{C}$ & \\
\hline $\begin{array}{l}\text { Benzathine } \\
\text { penicillin } G^{\star}\end{array}$ & Penicillins & B & 215 \\
\hline Cefaclor & Cephalosporins & A & \\
\hline Cefadroxil & Cephalosporins & A & \\
\hline Cefepime & Cephalosporins & A & \\
\hline Cefotaxime & Cephalosporins & A & \\
\hline Ceftazidime & Cephalosporins & $\mathrm{A}$ & \\
\hline Ceftriaxone & Cephalosporins & $\mathrm{A}$ & 280 \\
\hline Cefuroxime axetil & Cephalosporins & B & \\
\hline Cefalexin" & Cephalosporins & $\mathrm{A}$ & 230 \\
\hline Cefazolin & Cephalosporins & $\mathrm{A}$ & \\
\hline Cefradine & Cephalosporins & A & \\
\hline Ciprofloxacin & Quinolones & $\mathrm{C}$ & 280 \\
\hline Clarithromycin & Macrolides & $\mathrm{C}$ & \\
\hline Clavulanic acid & $\beta$-lactamase inhibitor & $\mathrm{A}$ & 230 \\
\hline Clindamycin & Macrolides & $\mathrm{A}$ & \\
\hline Cloxacillin & Penicillins & $\mathrm{A}$ & \\
\hline Doxycycline" & Tetracyclines & $\mathrm{A}$ & 350 \\
\hline Erythromycin ${ }^{1}$ & Macrolides & $\mathrm{B}$ & \\
\hline Gentamicin ${ }^{1}$ & Aminoglycosides & $\mathrm{C}$ & \\
\hline Griseofulvin * & Antifungal agent & B & 254 \\
\hline Lincomycin & Macrolides & A & \\
\hline Neomycin ${ }^{1}$ & Aminoglycosides & $\mathrm{C}$ & \\
\hline Nitrofurantoin & Nitrofurans & $\begin{array}{l}\text { Dimethylformamide } \\
\text { (DMF) }\end{array}$ & 254 \\
\hline Norfloxacin ${ }^{*}$ & Quinolones & $\mathrm{C}$ & 280 \\
\hline Ofloxacin" & Quinolones & $\mathrm{C}$ & 280 \\
\hline Penicillin $\mathrm{V}^{*}$ & Penicillins & A & 215 \\
\hline Polymyxin & Polypeptides & $\mathrm{A}$ & \\
\hline Rifampicin & Rifamycins & $\mathrm{B}$ & \\
\hline Roxithromycin" & Macrolides & $\mathrm{A}$ & 215 \\
\hline Sulfamethoxazole & Sulfonamides & $\mathrm{B}$ & 254 \\
\hline $\begin{array}{l}\text { Tetracycline } \\
(\mathrm{HCl})^{*}\end{array}$ & Tetracyclines & A & 280 \\
\hline Trimethoprim* & Other & B & 215 \\
\hline
\end{tabular}

* Compound used for validation.

${ }^{1}$ Compound only included in the screening method, not included in the quantification method.

\subsection{Sample preparation}

\subsubsection{Screening method}

2.3.1.1. Validation samples. Individual stock solutions $(1 \mathrm{mg} / \mathrm{mL})$ were prepared as described in Section 2.1.2. To build up a screening library with MS data, working solutions of $0.1 \mathrm{mg} / \mathrm{mL}$ were made by diluting individual stock solutions with the corresponding solvent (See Table 1).

To verify the matrix effect of capsules and tablets, three typical matrices were used. Matrix 1 was composed of talc and lactose, matrix 2 consisted of cellulose and starch and matrix 3 was made up of mannitol and croscarmellose sodium. Sucrose, maltodextrin, methylparaben and propylparaben were tested for the matrix effect of syrup. The antimicrobials were divided into three groups according to their corresponding solvents, namely methanol, methanol/ $\mathrm{H}_{2} \mathrm{O}$ mixture (50:50, v/ v) and methanol/ $\mathrm{H}_{2} \mathrm{O}$ with $1 \% \mathrm{HCOOH}$ mixture (20:80, v/v) (Table 1 ). The stock mixture solutions $(1 \mathrm{mg} / \mathrm{mL})$ of three groups were prepared respectively. $30 \mathrm{mg}$ of one of the selected matrices was brought into a flask of $10.0 \mathrm{~mL}$ and spiked with the appropriate amounts of stock mixture solutions of antimicrobials. The content of the flask was brought up to $10.0 \mathrm{~mL}$ to obtain a concentration of $25 \mu \mathrm{g} / \mathrm{mL}$ active ingredient.
2.3.1.2. Sample treatment. The number of tablets or capsules to be pulverized was determined as follows: i) if the number of offered units is less than or equal to 3 , the number to be pulverized is one, ii) if the number of offered units is more than 3 , but less than or equal to 11 , the number to be pulverized is half of the total unit number, iii) if the number of offered units is more than 11, the number to be pulverized is a quarter of the total unit number. The maximum number to be pulverized is 20. This way of working allows to preserve enough sample for retest or in case the inspection or the justice department does not want all of the evidence pieces to be destroyed.

An amount of $25 \mathrm{mg}$ of the pulverized drug products was accurately weighed and around $20 \mathrm{~mL}$ of the corresponding solvent was added, followed by ultrasonication for $15 \mathrm{~min}$. Next, the acquired solution was brought up to $25.0 \mathrm{~mL}$, further diluted 10 times and filtered through $0.2 \mu \mathrm{m}$ polytetrafluoroethylene (PTFE) filters before injection. A comparison between the use of filtration and centrifugation was executed to check whether some components were retained in the filters. The outcome indicated that there was no significant difference between the two debris removal techniques.

\subsubsection{Quantification method}

2.3.2.1. Calibration standards. The concentrations of the calibration standards are indicated in Table 2 and Table 3. Calibration standards were made by diluting the stock solution of each antimicrobial in the corresponding solvent as indicated in Table 1 . The solutions were filtered through $0.2 \mu \mathrm{m}$ PTFE filters before injection.

2.3.2.2. Validation samples. The placebo was made by mixing talc, lactose, cellulose, starch, mannitol and croscarmellose sodium in equal proportions. These compounds are the most commonly present excipients in tablets and capsules. The spiked placebo validation samples were prepared by adding a certain amount of individual stock solution to $30 \mathrm{mg}$ of placebo and diluting with the corresponding solvent to $10.0 \mathrm{~mL}$, in order to obtain the concentrations mentioned in Table 2. The solutions were ultrasonicated for $15 \mathrm{~min}$ and filtered through $0.2 \mu \mathrm{m}$ PTFE filters before injection.

To perform a recovery study, a mimic tablet was made by mixing the labelled amount of active pharmaceutical ingredient (API) with an appropriate amount of placebo according to the art of pharmacy profession. The added amount of placebo for each sample was calculated by subtracting the labelled API content from the average weight of a tablet. This simulated drug products underwent the same preparation procedure as described in Section 2.3.2.3. Each sample was analyzed in triplicate. Calibration ranges and recovery concentrations are shown in Table 3.

2.3.2.3. Sample treatment. An amount of $30 \mathrm{mg}$ of the pulverized drug products (Section 2.3.1.2) was accurately weighed and around $8 \mathrm{~mL}$ of the corresponding solvent was added, followed by ultrasonication for $15 \mathrm{~min}$. Then, the acquired solution was brought to $10.0 \mathrm{~mL}$. Further dilution was required to obtain an intermediate concentration within the calibration range. The solutions were filtered through $0.2 \mu \mathrm{m}$ PTFE filters before injection.

\subsection{Instrumental conditions}

\subsubsection{Screening method}

The screening of all samples was performed by UHPLC-MS ${ }^{2}$ on a Dionex UltiMate 3000 Rapid Separation LC (RSLC) system (Thermo Scientific, Sunnyvale, CA, USA) connected to an amaZon ${ }^{\mathrm{TM}}$ speed ETD mass spectrometer (Bruker Daltonics, Bremen, Germany). The chromatographic separation was carried out at $25^{\circ} \mathrm{C}$ on a Waters Acquity BEH shield RP18 $(150 \mathrm{~mm} \times 2.1 \mathrm{~mm}, 1.7 \mu \mathrm{m})$ column. The flow rate was $0.3 \mathrm{~mL} / \mathrm{min}$ and the injection volume was $2 \mu \mathrm{L}$. Mobile phases A and $\mathrm{B}$ consisted of $0.1 \%$ formic acid in water and $0.1 \%$ formic acid in 
Table 2

Concentrations of the calibration standards and the validation samples.

\begin{tabular}{|c|c|c|}
\hline Compounds & $\begin{array}{l}\text { Concentrations } \\
\text { Calibration standards } \\
(\mu \mathrm{g} / \mathrm{mL})\end{array}$ & $\begin{array}{l}\text { Validation samples } \\
(\mu \mathrm{g} / \mathrm{mL})\end{array}$ \\
\hline \multirow[t]{4}{*}{ Amoxicillin } & 10 & 30 \\
\hline & 100 & 150 \\
\hline & 200 & 300 \\
\hline & 400 & \\
\hline \multirow[t]{4}{*}{ Ampicillin } & 10 & 30 \\
\hline & 100 & 150 \\
\hline & 200 & 300 \\
\hline & 400 & \\
\hline \multirow[t]{4}{*}{ Benzathine penicillin G } & 10 & 30 \\
\hline & 50 & 90 \\
\hline & 100 & 150 \\
\hline & 200 & \\
\hline \multirow[t]{4}{*}{ Clavulanic acid } & 10 & 10 \\
\hline & 20 & 30 \\
\hline & 40 & 50 \\
\hline & 60 & \\
\hline \multirow[t]{4}{*}{ Ceftriaxone } & 150 & 150 \\
\hline & 200 & 200 \\
\hline & 300 & 300 \\
\hline & 350 & \\
\hline \multirow[t]{4}{*}{ Cefalexin } & 10 & 30 \\
\hline & 100 & 150 \\
\hline & 200 & 300 \\
\hline & 400 & \\
\hline \multirow[t]{4}{*}{ Ciprofloxacin } & 10 & 30 \\
\hline & 50 & 90 \\
\hline & 100 & 150 \\
\hline & 150 & \\
\hline \multirow[t]{4}{*}{ Doxycycline } & 10 & 30 \\
\hline & 100 & 150 \\
\hline & 200 & 300 \\
\hline & 400 & \\
\hline \multirow[t]{4}{*}{ Ofloxacin } & 10 & 30 \\
\hline & 100 & 150 \\
\hline & 200 & 300 \\
\hline & 400 & \\
\hline \multirow[t]{4}{*}{ Penicillin V } & 10 & 30 \\
\hline & 100 & 150 \\
\hline & 200 & 300 \\
\hline & 400 & \\
\hline \multirow[t]{4}{*}{ Roxithromycin } & 10 & 30 \\
\hline & 100 & 150 \\
\hline & 200 & 300 \\
\hline & 350 & \\
\hline \multirow[t]{4}{*}{ Tetracycline (HCl) } & 10 & 30 \\
\hline & 100 & 150 \\
\hline & 200 & 300 \\
\hline & 400 & \\
\hline
\end{tabular}

Table 3

Recoveries of five additionally investigated antimicrobials (SD: standard deviation).

\begin{tabular}{lllr}
\hline Compounds & $\begin{array}{l}\text { Concentrations } \\
\text { Calibration } \\
\text { standards }(\mu \mathrm{g} / \mathrm{mL})\end{array}$ & $\begin{array}{l}\text { Recovery concentrations } \\
(\mu \mathrm{g} / \mathrm{mL})\end{array}$ & $\begin{array}{l}\text { Recovery: } \\
\text { mean \% (SD) }\end{array}$ \\
\hline Griseofulvin & $10,50,100,200$ & 84 & $99.6(1.4)$ \\
Norfloxacin & $10,50,100,200$ & 102 & $98.9(1.0)$ \\
Nitrofurantoin & $10,50,100,200$ & 96 & $98.9(1.2)$ \\
Sulfamethoxazole & $10,50,100,200$ & 119 & $102.9(0.7)$ \\
Trimethoprim & $10,100,200,400$ & 238 & $98.0(0.2)$ \\
\hline
\end{tabular}

ACN, respectively. The chromatographic gradient started with an isocratic elution of $99 \%$ A for $1 \mathrm{~min}$, followed by a linear decrease to $85 \%$ in $4 \mathrm{~min}$, then the percent of mobile phase A further linearly dropped to $25 \%$ in 10 min, after which the column was rinsed by $1 \% \mathrm{~A}$ for $1.5 \mathrm{~min}$. The post-gradient equilibration with $99 \%$ A was performed for $1.5 \mathrm{~min}$.
The total run time was $18 \mathrm{~min}$ for the screening method.

The mass spectrometer settings were based on those described by Vanhee et al. [20]: the mass spectrometer was operated in alternating positive electrospray ionization (ESI + ) and negative electrospray ionization (ESI-) mode, with a spray voltage of $3.5 \mathrm{kV}$ and an end plate voltage of $500 \mathrm{~V}$. The nebulizer was set to $2 \mathrm{bar}$ and the desolvation gas temperature was $180^{\circ} \mathrm{C}$ at a flow rate of $4.0 \mathrm{~L} / \mathrm{min}$. MS spectra were obtained within a mass range of $100-1000 \mathrm{~m} / \mathrm{z}$ and the smart parameter setting (SPS) was set to $475 \mathrm{~m} / z$. For MS ${ }^{2}$ precursor selection, the most intense ion was isolated above an absolute intensity of 2.500 and a $5 \%$ relative intensity threshold. The ion charge control (ICC) was set to 100,000 for ESI- and 200,000 for ESI + with a maximum accumulation time of $200 \mathrm{~ms}$. CID (Collision Induced Dissociation) was performed using helium as collision gas. The fragmentation amplitude was set to $100 \%$ using SmartFrag ${ }^{\mathrm{TM}}$ Enhanced for amplitude ramping (80-120\%). The fragmentation time was set to $20 \mathrm{~ms}$.

\subsubsection{Quantification method}

Method development, optimization, validation and application were achieved on an Aquity UPLC system (Waters, Milford, MA, USA) equipped with a binary solvent manager, a sample manager and a DAD detector. Data integration and analysis were performed with Empower 3 Citrix software.

The optimal separation (see also Section 3.1 ) was obtained on a Waters Acquity BEH shield RP18 $(150 \mathrm{~mm} \times 2.1 \mathrm{~mm}, 1.7 \mu \mathrm{m})$ column, which is the same as the screening method. The column and sample temperatures were kept at $25{ }^{\circ} \mathrm{C}$ and $15^{\circ} \mathrm{C}$, respectively. The flow rate was $0.2 \mathrm{~mL} / \mathrm{min}$ and the injection volume was $5 \mu \mathrm{L}$. The gradient was composed of $0.01 \%$ formic acid in water as mobile phase A and ACN as mobile phase B. The gradient started with an isocratic elution of $99 \%$ A for $5 \mathrm{~min}$ followed by a linear decrease to $85 \%$ in $10 \mathrm{~min}$. Then the percent of mobile phase A further linearly dropped to $25 \%$ in $15 \mathrm{~min}$, followed by returning to the initial composition in $2 \mathrm{~min}$. A wavelength scan with the DAD detector was performed for all antimicrobials within the range $190-400 \mathrm{~nm}$ for identity confirmation. For method development and optimization, all antimicrobials were detected and monitored at $215 \mathrm{~nm}$ whereas the method validation and application were performed at a corresponding detection wavelength for each antimicrobial as shown in Table 1.

\subsection{Method validation}

\subsubsection{Screening method}

The screening method should avoid false positive and false negative results as much as possible. Therefore, it has to be validated in terms of sensitivity, selectivity and matrix effects. The evaluation of sensitivity is based on the measurement of the screening detection limit (SDL), defined as the lowest concentration at which an analyte can be correctly detected in at least $95 \%$ of the samples $[21,22]$. The selectivity ensures the method to detect the analyte of interest without interferences of other analytes and excipients. Blank samples (only matrix) were tested to validate false detects. The detection of the active compounds was verified in the presence of different matrices as described in Section 2.3.1.1. According to "Method validation and quality control procedures for pesticide residues analysis in food and feed" (Sanco/12495/ 2011) [21], the validation should include at least 20 samples covering multiple matrices with a minimum of two samples per matrix. In the present study, 36 samples covering three matrices, representative for the matrix scope of the laboratory, were analyzed before and after spiking, in which the concentration was in line with a SDL of $25 \mu \mathrm{g} / \mathrm{mL}$ (See Section 3.3). To verify the matrix effect of syrup, the retention times of sucrose, maltodextrin, methylparaben and propylparaben were determined to check interferences [21].

\subsubsection{Quantification method}

Analytical method validation aims to assure that future 


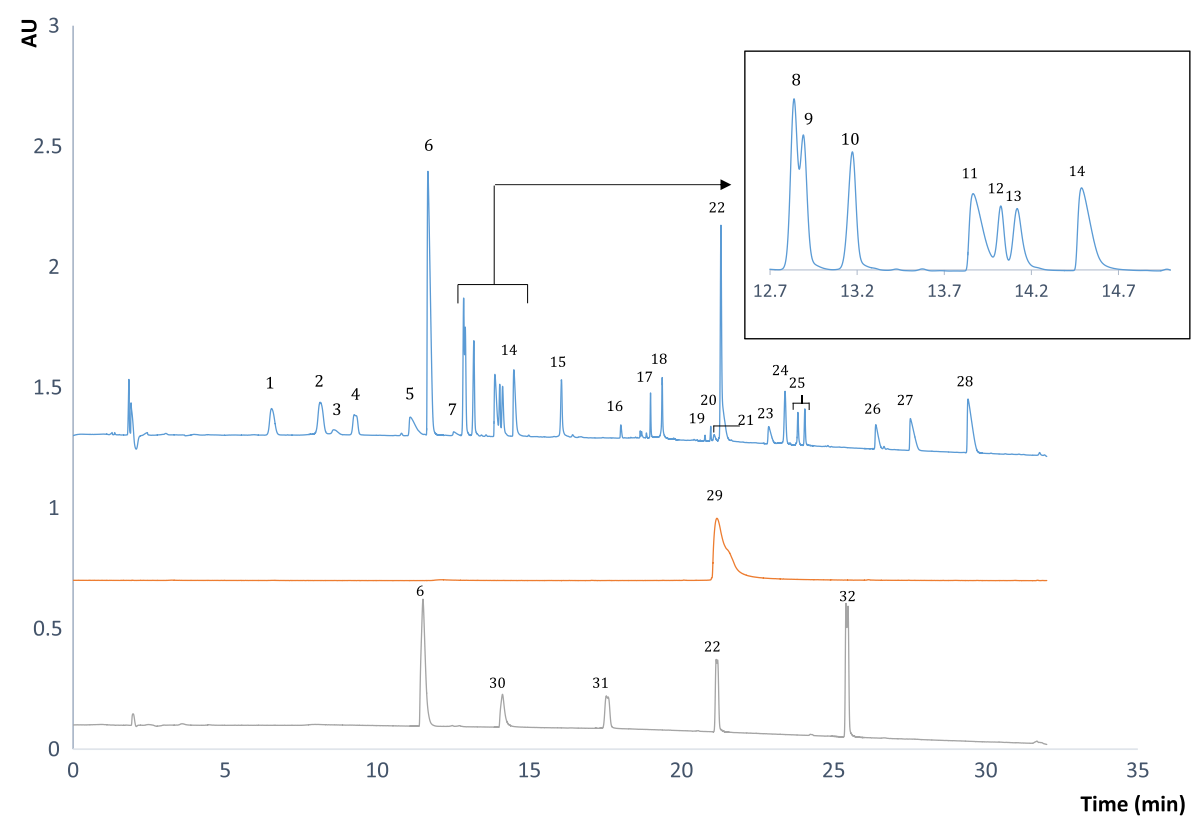

Fig. 1. Typical chromatograms obtained by the UHPLC-DAD quantification method $(1=$ amoxicillin, $2=$ cefadroxil,$\quad 3$ = lincomycin,$\quad 4=$ cefepime, $5=$ clavulanic acid, $6=$ trimethoprim, $7=$ polymyxin B1, $8=$ cefalexin, $9=$ ampicillin, $10=$ cefaclor, $11=$ ofloxacin, $12=$ cefradine, $13=$ ceftazidime, $\quad 14=$ ciprofloxacin, $\quad 15=$ tetracycline, $16=$ clindamycin, $17=$ bacitracin $\mathrm{Zn}, 18=$ doxycycline, $\quad 19=$ clarithromycin,$\quad 20=$ roxithromycin, 21 = cefotaxime, 22 = sulfamethoxazole, $23=$ cefazolin, 24 = rifampicin, $25=$ cefuroxime axetil, 26 = benzathine penicillin G, $27=$ penicillin $\mathrm{V}$, $28=$ cloxacillin, $29=$ ceftriaxone, $30=$ norfloxacin, $31=$ nitrofurantoin, $32=$ griseofulvin).

measurements for the content of analytes are reliable and close enough to the true value. The UHPLC-DAD quantification method was validated according to ISO-17025 via the "total error" approach by building accuracy profiles, a graphical decision-making tool $[23,24]$. It is a fitnessfor-purpose approach, which evaluates the systematic error (trueness) as well as the random error (intermediate precision) to know the deviation between the measured result and the true value. This approach is able to compute an interval predicting the values of future measurements.

Specifically, the "total error" approach verifies whether the analytical method could provide a result that deviates from the true value within the predefined acceptability limits $[-\lambda, \lambda]$, being for licensed pharmaceutical products $[-5 \%, 5 \%]$. This is carried out by the calculation of the $\beta$-expectation tolerance interval, which comprises the future results that will fall inside the acceptability limits with a given probability $\beta[25,26]$. In this study, $\beta$ was set at $95 \%$ and the calculation was made at three concentration levels. The method can be regarded as accurate within the studied concentration range on the premise that the calculated $\beta$-expectation tolerance interval is located within the acceptance limits of $\pm 5 \%$ [25].

In practice, the quantification method was only fully validated for the 11 antibiotics (amoxicillin, ampicillin, benzathine penicillin G, ceftriaxone, cefalexin, ciprofloxacin, doxycycline, ofloxacin, penicillin $\mathrm{V}$, roxithromycin, tetracycline) and 1 beta-lactamase inhibitor (clavulanic acid) that were present from the beginning and for which positive hits were obtained in the screening experiment. Validation samples at three different concentration levels were prepared daily as three independent replicates (See Table 2). The analysis of validation samples continued for at least three days depending on the compounds. The concentrations were determined by back-calculation based on the calibration lines (see Section 2.3.2.1). Based on these calculated concentrations, the linearity of the results, trueness, precision (repeatability and intermediate precision) and accuracy were assessed [27].

Since the samples of five other antimicrobials (nitrofurantoin, norfloxacin, trimethoprim, sulfamethoxazole and griseofulvin) were received at a later stage, a reduced validation of the quantification method was performed where the construction of accuracy profiles was replaced by recovery tests.

\section{Results and discussion}

\subsection{Quantification method development and optimization}

Because MS is less cost-effective than $\mathrm{DAD}$, the quantification method was developed first starting with the set of 35 antimicrobials and 1 beta-lactamase inhibitor (Section 2.2). However, erythromycin, azithromycin, neomycin and gentamicin had to be excluded due to their insufficient UV absorbance in combination with the selected mobile phases. A Waters Acquity BEH shield RP18 column $(150 \mathrm{~mm} \times 2.1 \mathrm{~mm}$, $1.7 \mu \mathrm{m}$ ) was used to start the method development, because many reports have demonstrated that it can generate a satisfactory separation among antibiotics such as quinolones, macrolides and $\beta$-lactams [28-30]. ACN was initially used as solvent for all standards.

Concerning the mobile phases, $0.01 \%$ formic acid in water and ACN were selected. ACN was chosen as organic modifier due to its low cutoff value and more stable baseline compared to methanol. The initial UHPLC gradient started with $95 \%$ aqueous phase, which was held for $5 \mathrm{~min}$ followed by a linear decrease to $5 \%$ in $30 \mathrm{~min}$ and an isocratic elution for $10 \mathrm{~min}$ before returning to the initial conditions in $10 \mathrm{~min}$. Flow rates of $0.2 \mathrm{~mL} / \mathrm{min}, 0.3 \mathrm{~mL} / \mathrm{min}$ and $0.4 \mathrm{~mL} / \mathrm{min}$ were tested as well as column temperatures of $25^{\circ} \mathrm{C}, 30^{\circ} \mathrm{C}$ and $40^{\circ} \mathrm{C}$. It was found that the flow rate of $0.2 \mathrm{~mL} / \mathrm{min}$ and the column temperature of $25^{\circ} \mathrm{C}$ gave the best performance in general. Since the separation among cefadroxil, lincomycin and cefepime was not optimal, the starting condition of the gradient was changed from $5 \%$ ACN to $1 \%$ ACN. However, some peak shapes were not ideal and even distorted. This problem was solved by using methanol as solvent instead of ACN. In order to shorten the analysis time, the percentage of ACN was calculated at the moment the last peak eluted. In this way, the gradient could be stopped at 75\% ACN instead of $95 \%$. Under these conditions, cefalexin and ampicillin coeluted, but this was solved by decreasing the slope of the increase rate of ACN in the elution time frame of 5-15 min. Under the final conditions mentioned in Section 2.4.2, all 32 compounds were separated. Typical chromatograms obtained with the references of all 31 antimicrobials and the 1 beta-lactamase inhibitor are presented in Fig. 1.

For the quantification of erythromycin, azithromycin, neomycin and gentamicin, the chromatographic procedures in the respective monographs of the European Pharmacopoeia [18] can be followed. 


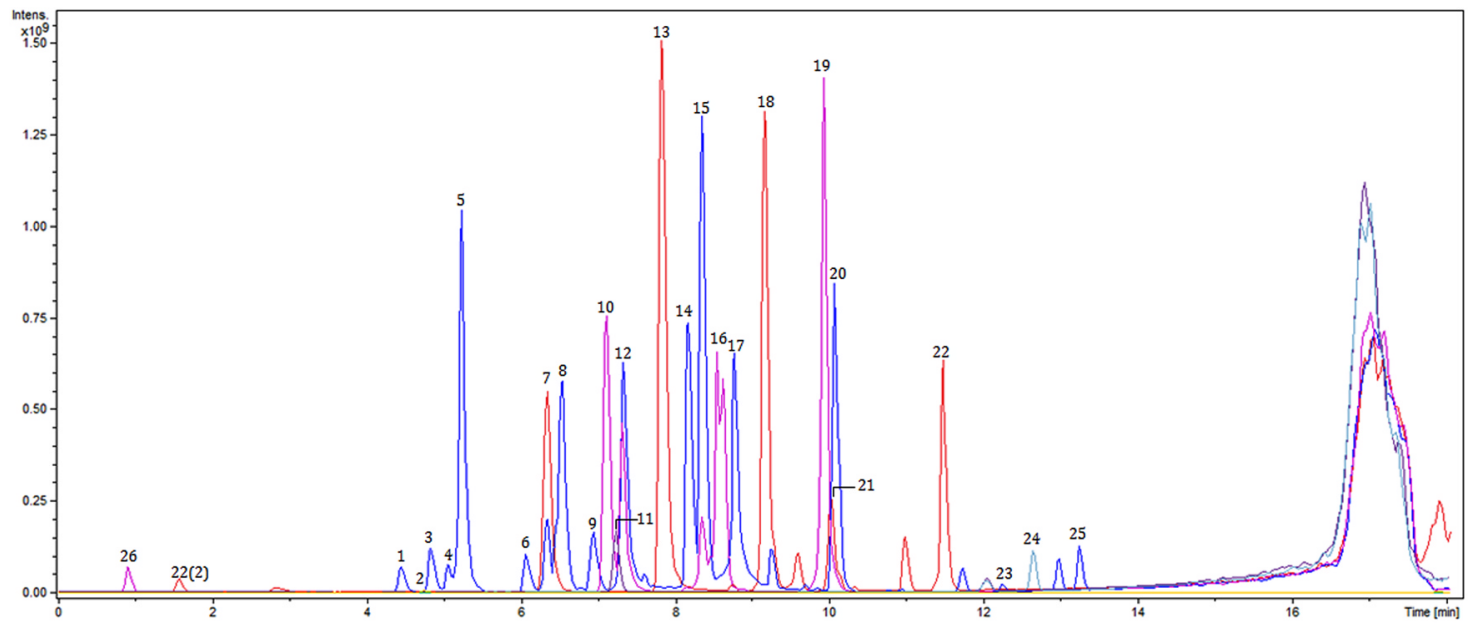

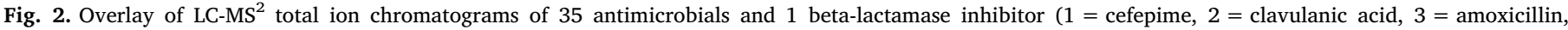

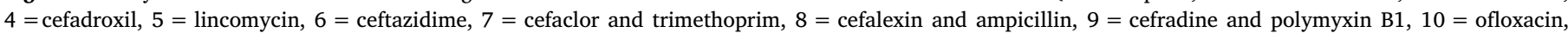

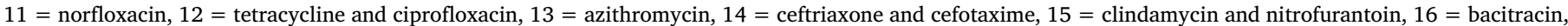

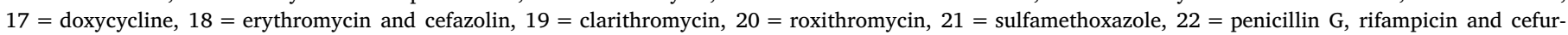
oxime axetil, 22(2) = benzathine (of benzathine penicillin $\mathrm{G}$ ), $23=$ penicillin $\mathrm{V}, 24=$ griseofulvin, $25=$ cloxacillin, $26=$ gentamicin and neomycin).

Table 4

Characteristics of antimicrobials by LC-MS ${ }^{2}(\mathrm{~m} / \mathrm{z}$ : mass-to-charge ratio).

\begin{tabular}{|c|c|c|c|c|c|c|}
\hline Name & Monoisotopic mass (Da) & Chemical formula & Retention time & $\mathrm{LOD}^{*}(\mu \mathrm{g} / \mathrm{mL})$ & Adduct & $m / z$ \\
\hline Amoxicillin & 365.10 & $\mathrm{C}_{16} \mathrm{H}_{19} \mathrm{~N}_{3} \mathrm{O}_{5} \mathrm{~S}$ & 5.0 & 2.5 & {$[\mathrm{M}+\mathrm{H}]^{+}$} & 366.1 \\
\hline Ampicillin & 349.11 & $\mathrm{C}_{16} \mathrm{H}_{19} \mathrm{~N}_{3} \mathrm{O}_{4} \mathrm{~S}$ & 6.6 & 1 & {$[\mathrm{M}+\mathrm{H}]^{+}$} & 350.1 \\
\hline Azithromycin & 748.51 & $\mathrm{C}_{38} \mathrm{H}_{72} \mathrm{~N}_{2} \mathrm{O}_{12}$ & 8.1 & 1 & {$[\mathrm{M}+2 \mathrm{H}]^{2+}$} & 375.2 \\
\hline Bacitracin & 1421.75 & $\mathrm{C}_{66} \mathrm{H}_{103} \mathrm{~N}_{17} \mathrm{O}_{16} \mathrm{~S}$ & 8.7 & 1 & {$[\mathrm{M}+3 \mathrm{H}]^{3+}$} & 475.0 \\
\hline Benzathine & 240.16 & $\mathrm{C}_{16} \mathrm{H}_{20} \mathrm{~N}_{2}$ & 2.0 & 10 & {$[\mathrm{M}+\mathrm{H}]^{+}$} & 241.0 \\
\hline Benzylpenicillin & 334.10 & $\mathrm{C}_{16} \mathrm{H}_{18} \mathrm{~N}_{2} \mathrm{O}_{4} \mathrm{~S}$ & 11.2 & 0.25 & {$[\mathrm{M}+\mathrm{H}]^{+}$} & 335.1 \\
\hline Cefaclor & 367.04 & $\mathrm{C}_{15} \mathrm{H}_{14} \mathrm{ClN}_{3} \mathrm{O}_{4} \mathrm{~S}$ & 6.4 & 1 & {$[\mathrm{M}+\mathrm{H}]^{+}$} & 368.0 \\
\hline Cefadroxil & 363.09 & $\mathrm{C}_{16} \mathrm{H}_{17} \mathrm{~N}_{3} \mathrm{O}_{5} \mathrm{~S}$ & 5.2 & 1 & {$[\mathrm{M}+\mathrm{H}]^{+}$} & 364.0 \\
\hline Cefepime & 480.13 & $\mathrm{C}_{19} \mathrm{H}_{24} \mathrm{~N}_{6} \mathrm{O}_{5} \mathrm{~S}_{2}$ & 4.6 & 5 & {$[\mathrm{M}+\mathrm{H}]^{+}$} & 481.1 \\
\hline Cefotaxime & 455.06 & $\mathrm{C}_{16} \mathrm{H}_{17} \mathrm{~N}_{5} \mathrm{O}_{7} \mathrm{~S}_{2}$ & 8.2 & 1 & {$[\mathrm{M}+\mathrm{H}]^{+}$} & 456.0 \\
\hline Ceftazidime & 546.10 & $\mathrm{C}_{22} \mathrm{H}_{22} \mathrm{~N}_{6} \mathrm{O}_{7} \mathrm{~S}_{2}$ & 6.1 & 2.5 & {$[\mathrm{M}+2 \mathrm{H}]^{2+}$} & 274.0 \\
\hline Ceftriaxone & 554.05 & $\mathrm{C}_{18} \mathrm{H}_{18} \mathrm{~N}_{8} \mathrm{O}_{7} \mathrm{~S}_{3}$ & 7.9 & 25 & {$[\mathrm{M}+\mathrm{H}]^{+}$} & 555.0 \\
\hline Cefuroxime axetil & 510.11 & $\mathrm{C}_{20} \mathrm{H}_{22} \mathrm{~N}_{4} \mathrm{O}_{10} \mathrm{~S}$ & $11.7 / 12.3$ & 1 & {$[\mathrm{M}+\mathrm{Na}]^{+}$} & 533.1 \\
\hline Cefalexin & 347.09 & $\mathrm{C}_{16} \mathrm{H}_{17} \mathrm{~N}_{3} \mathrm{O}_{4} \mathrm{~S}$ & 6.6 & 2.5 & {$[\mathrm{M}+\mathrm{H}]^{+}$} & 348.1 \\
\hline Cefazolin & 454.03 & $\mathrm{C}_{14} \mathrm{H}_{14} \mathrm{~N}_{8} \mathrm{O}_{4} \mathrm{~S}_{3}$ & 9.2 & 5 & {$[\mathrm{M}+\mathrm{H}]^{+}$} & 455.0 \\
\hline Cefradine & 349.11 & $\mathrm{C}_{16} \mathrm{H}_{19} \mathrm{~N}_{3} \mathrm{O}_{4} \mathrm{~S}$ & 7.1 & 1 & {$[\mathrm{M}+\mathrm{H}]^{+}$} & 350.1 \\
\hline Ciprofloxacin & 331.13 & $\mathrm{C}_{17} \mathrm{H}_{18} \mathrm{FN}_{3} \mathrm{O}_{3}$ & 7.4 & 0.25 & {$[\mathrm{M}+\mathrm{H}]^{+}$} & 332.1 \\
\hline Clarithromycin & 747.48 & $\mathrm{C}_{38} \mathrm{H}_{69} \mathrm{NO}_{13}$ & 10.2 & 0.1 & {$[\mathrm{M}+\mathrm{H}]^{+}$} & 748.5 \\
\hline Clavulanic acid & 199.05 & $\mathrm{C}_{8} \mathrm{H}_{9} \mathrm{NO}_{5}$ & 4.7 & 15 & {$[2 \mathrm{M}-\mathrm{H}]^{-}$} & 396.8 \\
\hline Clindamycin & 424.18 & $\mathrm{C}_{18} \mathrm{H}_{33} \mathrm{ClN}_{2} \mathrm{O}_{5} \mathrm{~S}$ & 8.5 & 0.1 & {$[\mathrm{M}+\mathrm{H}]^{+}$} & 425.2 \\
\hline Cloxacillin & 435.07 & $\mathrm{C}_{19} \mathrm{H}_{18} \mathrm{ClN}_{3} \mathrm{O}_{5} \mathrm{~S}$ & 13.2 & 1 & {$[\mathrm{M}+\mathrm{H}]^{+}$} & 436.0 \\
\hline Doxycycline & 444.15 & $\mathrm{C}_{22} \mathrm{H}_{24} \mathrm{~N}_{2} \mathrm{O}_{8}$ & 8.9 & 0.1 & {$[\mathrm{M}+\mathrm{H}]^{+}$} & 445.1 \\
\hline Erythromycin A & 733.46 & $\mathrm{C}_{37} \mathrm{H}_{67} \mathrm{NO}_{13}$ & 9.5 & 0.5 & {$[\mathrm{M}+\mathrm{H}]^{+}$} & 734.5 \\
\hline Gentamicin & 477.32 & $\mathrm{C}_{21} \mathrm{H}_{43} \mathrm{~N}_{5} \mathrm{O}_{7}$ & 1.0 & 10 & {$[\mathrm{M}+\mathrm{H}]^{+}$} & 478.3 \\
\hline Griseofulvin & 352.07 & $\mathrm{C}_{17} \mathrm{H}_{17} \mathrm{ClO}_{6}$ & 12.6 & 1 & {$[\mathrm{M}+\mathrm{H}]^{+}$} & 353.0 \\
\hline Lincomycin & 406.21 & $\mathrm{C}_{18} \mathrm{H}_{34} \mathrm{~N}_{2} \mathrm{O}_{6} \mathrm{~S}$ & 5.4 & 0.1 & {$[\mathrm{M}+\mathrm{H}]^{+}$} & 407.2 \\
\hline Neomycin & 614.31 & $\mathrm{C}_{23} \mathrm{H}_{46} \mathrm{~N}_{6} \mathrm{O}_{13}$ & 0.9 & 10 & {$[\mathrm{M}+\mathrm{H}]^{+}$} & 615.3 \\
\hline Nitrofurantoin & 238.03 & $\mathrm{C}_{8} \mathrm{H}_{6} \mathrm{~N}_{4} \mathrm{O}_{5}$ & 8.3 & 5 & {$[\mathrm{M}-\mathrm{H}]^{-}$} & 236.8 \\
\hline Norfloxacin & 319.13 & $\mathrm{C}_{16} \mathrm{H}_{18} \mathrm{FN}_{3} \mathrm{O}_{3}$ & 7.2 & 1 & {$[\mathrm{M}+\mathrm{H}]^{+}$} & 320.0 \\
\hline Ofloxacin & 361.14 & $\mathrm{C}_{18} \mathrm{H}_{20} \mathrm{FN}_{3} \mathrm{O}_{4}$ & 7.2 & 0.1 & {$[\mathrm{M}+\mathrm{H}]^{+}$} & 362.1 \\
\hline Penicillin V & 350.09 & $\mathrm{C}_{16} \mathrm{H}_{18} \mathrm{~N}_{2} \mathrm{O}_{5} \mathrm{~S}$ & 12.3 & 2.5 & {$[2 \mathrm{M}-\mathrm{H}]^{-}$} & 699.3 \\
\hline Polymyxin B1 & 1202.75 & $\mathrm{C}_{56} \mathrm{H}_{98} \mathrm{~N}_{16} \mathrm{O}_{13}$ & 6.9 & 25 & {$[\mathrm{M}+3 \mathrm{H}]^{3+}$} & 402.0 \\
\hline Rifampicin & 822.41 & $\mathrm{C}_{43} \mathrm{H}_{58} \mathrm{~N}_{4} \mathrm{O}_{12}$ & 11.8 & 0.25 & {$[\mathrm{M}+\mathrm{H}]^{+}$} & 823.4 \\
\hline Roxithromycin & 836.52 & $\mathrm{C}_{41} \mathrm{H}_{76} \mathrm{~N}_{2} \mathrm{O}_{15}$ & 10.3 & 0.1 & {$[\mathrm{M}+\mathrm{H}]^{+}$} & 837.5 \\
\hline Sulfamethoxazole & 253.05 & $\mathrm{C}_{10} \mathrm{H}_{11} \mathrm{~N}_{3} \mathrm{O}_{3} \mathrm{~S}$ & 10.2 & 0.5 & {$[\mathrm{M}+\mathrm{H}]^{+}$} & 253.9 \\
\hline Tetracycline & 444.15 & $\mathrm{C}_{22} \mathrm{H}_{24} \mathrm{~N}_{2} \mathrm{O}_{8}$ & 7.5 & 0.1 & {$[\mathrm{M}+\mathrm{H}]^{+}$} & 445.1 \\
\hline Trimethoprim & 290.14 & $\mathrm{C}_{14} \mathrm{H}_{18} \mathrm{~N}_{4} \mathrm{O}_{3}$ & 6.5 & 0.1 & {$[\mathrm{M}+\mathrm{H}]^{+}$} & 291.0 \\
\hline
\end{tabular}

* LOD was measured in the total ion chromatogram (TIC).

\subsection{Screening method development and optimization}

The screening method was developed and optimized based on the quantification method. By applying the mobile phases of the quantification method (A: $0.01 \%$ formic acid in water and B: ACN), ceftriaxone $(100 \mu \mathrm{g} / \mathrm{mL})$ could not be detected by MS. In order to acquire sufficient ionization and higher sensitivity, the mobile phases have been modified to $0.1 \%$ formic acid in water as mobile phase $\mathrm{A}$ and 


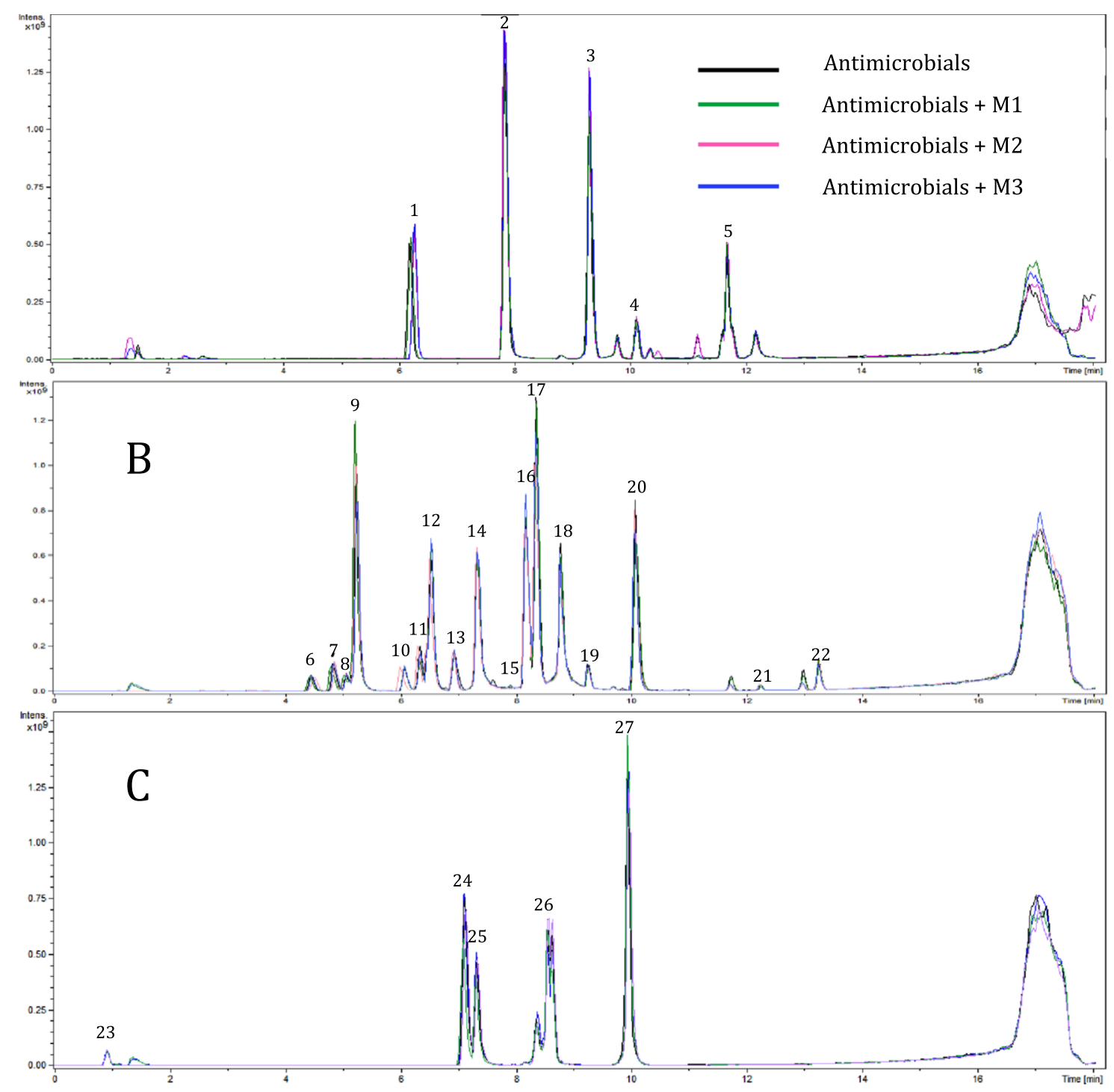

Fig. 3. Overlay of UHPLC-MS ${ }^{2}$ total ion chromatograms in the positive mode of antimicrobials in their corresponding solvent. A: the group of compounds dissolved in methanol, B in methanol/ $\mathrm{H}_{2} \mathrm{O}$ mixture $(50: 50, \mathrm{v} / \mathrm{v}), \mathrm{C}$ in methanol $/ \mathrm{H}_{2} \mathrm{O}$ with $1 \% \mathrm{HCOOH}$ mixture $(20: 80$, v/v) $(1=$ trimethoprim, $2=$ azithromycin, $3=$ erythromycin, 4 = sulfamethoxazole, $5=$ penicillin $\mathrm{G}$, cefuroxime axetil and rifampicin, $5(2)=$ benzathine (of benzathine penicillin $\mathrm{G}$ ), $6=$ cefepime, $7=$ amoxicillin, $8=$ cefadroxil, $9=$ lincomycin, $10=$ ceftazidime, $11=$ cefaclor, $12=$ cefalexin and ampicillin, $13=$ cefradine and polymyxin B1, $14=$ tetracycline, $15=$ ceftriaxone, $16=$ cefotaxime, $17=$ clindamycin, $18=$ doxycycline, $19=$ cefazolin, $20=$ roxithromycin, $21=$ penicillin $\mathrm{V}, 22=$ cloxacillin, $23=$ gentamicin and neomycin, 24 = ofloxacin, 25 = ciprofloxacin, 26 = bacitracin, 27 = clarithromycin, clavulanic acid was monitored in the negative mode).

$0.1 \%$ formic acid in ACN as mobile phase B. In this case, all 36 compounds could be detected. The injection volume was decreased from $5 \mu \mathrm{L}$ to $2 \mu \mathrm{L}$ due to higher sensitivity of the MS detector compared to the DAD detector. The column temperature was kept at $25^{\circ} \mathrm{C}$. To optimize the screening method, the flow rate was increased from $0.2 \mathrm{~mL} / \mathrm{min}$ to $0.3 \mathrm{~mL} / \mathrm{min}$. The LC gradient of the quantification method was further refined for a shorter analysis time which should be achievable due to the additional selectivity of MS. Moreover, the elution profiles of the different antimicrobials were not altered too much so that the link was kept between the screening and the quantification method. The initial isocratic elution of $99 \%$ A lasted for $1 \mathrm{~min}$ instead of $5 \mathrm{~min}$, then $99 \% \mathrm{~A}$ linearly decreased to $85 \%$ within $4 \mathrm{~min}$ and further decreased to $25 \%$ within $10 \mathrm{~min}$. Subsequently, online column cleaning with $1 \%$ mobile phase A was maintained for $1.5 \mathrm{~min}$. The total runtime decreased from $32 \mathrm{~min}$ to $18 \mathrm{~min}$ (including washing and re-equilibration). A typical LC-MS $^{2}$ total ion chromatogram obtained by the optimized method is shown in Fig. 2.

\subsection{Validation of the screening method}

\subsubsection{Sensitivity}

The sensitivity was evaluated based on the SDL, which was measured by the maximum value of the limit of detection (LOD) as explained in Section 3.4.2 among all compounds. The LOD values of all compounds were determined in the total ion chromatogram (TIC) and are presented in Table 4 . The SDL was set at $25 \mu \mathrm{g} / \mathrm{mL}$ for the necessary sensitivity of the screening method.

\subsubsection{Selectivity and matrix effect}

The selectivity was confirmed by the retention time of each antimicrobial and their corresponding MS spectra. A 0.3 Da tolerance on the MS and the $\mathrm{MS}^{2}$ spectra was considered acceptable. An in-house LC$\mathrm{MS}^{2}$ screening library was constructed by analysis of working solutions $(0.1 \mathrm{mg} / \mathrm{mL})$. The screening library comprised the theoretical monoisotopic exact mass, the mass-to-charge ratio $(\mathrm{m} / \mathrm{z})$ of the adduct with the most intense signal for each compound (shown in Table 4), the MS spectrum and the $\mathrm{MS}^{2}$ spectrum. Compounds eluting at the same time 
Table 5

Validation parameters of 17 compounds for the UHPLC-DAD quantification method (QC: quality coefficient).

\begin{tabular}{lccccc}
\hline Compounds & $\begin{array}{l}\text { LOD } \\
(\mu \mathrm{g} / \mathrm{mL})\end{array}$ & $\begin{array}{l}\text { LOQ } \\
(\mu \mathrm{g} / \mathrm{mL})\end{array}$ & Lack of fit & $\mathrm{R}^{2}$ & QC (\%) \\
\hline Amoxicillin & 0.1 & 0.5 & 0.7445 & 0.9997 & 1.455 \\
Ampicillin & 0.1 & 0.4 & 0.7999 & 0.9996 & 1.816 \\
Benzathine penicillin G & 0.2 & 0.8 & 0.0957 & 0.9994 & 2.024 \\
Ceftriaxone & 30 & 100 & 0.1901 & 0.9991 & 1.545 \\
Cefalexin & 0.1 & 0.3 & 0.9752 & 0.9997 & 1.605 \\
Ciprofloxacin & 0.2 & 0.5 & 0.0807 & 0.9996 & 1.428 \\
Clavulanic acid & 0.2 & 0.6 & 0.1405 & 0.9993 & 1.686 \\
Doxycycline & 2.5 & 8.0 & 0.0596 & 0.9993 & 2.334 \\
Ofloxacin & 0.2 & 0.4 & 0.8658 & 0.9994 & 2.069 \\
Penicillin V & 0.5 & 1.5 & 0.2064 & 0.9997 & 1.432 \\
Roxithromycin & 2.0 & 7.0 & 0.1405 & 0.9998 & 1.259 \\
Tetracycline (HCl) & 0.5 & 2.0 & 0.0698 & 0.9992 & 2.495 \\
Sulfamethoxazole & 0.1 & 0.3 & 0.1813 & 0.9994 & 2.116 \\
Trimethoprim & 1.0 & 2.5 & 0.2232 & 0.9997 & 1.639 \\
Nitrofurantoin & 0.1 & 0.3 & 0.0557 & 0.9994 & 2.116 \\
Norfloxacin & 0.1 & 0.3 & 0.1883 & 0.9998 & 1.274 \\
Griseofulvin & 0.5 & 2.0 & 0.1017 & 0.9999 & 0.655 \\
\hline
\end{tabular}

could be distinguished based on their different MS data. Additionally, 21 negative samples (containing only matrix components) and 36 samples (composed of matrix components spiked with the different antimicrobials) were analyzed. No false positives or false negatives could be observed, i.e. no antimicrobials were detected in the blank samples, while all targeted antimicrobials could be found in the spiked samples at a SDL level.

Overlay of total ion chromatograms of the solutions of antimicrobials and three matrices spiked with antimicrobials are shown in Fig. 3. It could be proved that no ion suppression occurred and that the matrices had a limited influence on the retention times (all shifts being less than $0.3 \mathrm{~min}$ ). As for the matrix effect of syrup, methylparaben showed no interference with any antimicrobial. However, sucrose and maltodextrin had the same retention time as benzathine penicillin G, and the same was found for propylparaben and cloxacillin. Even though benzathine penicillin $\mathrm{G}$ and cloxacillin co-eluted with syrup excipients, they had different MS and MS ${ }^{2}$ spectra. To conclude, the compound can be identified if it meets the screening criteria, which are: the shift of the retention time is less than or equal to $0.5 \mathrm{~min}$ (compared with the reference standard), $m / z$ of the mother ion is equal to this in the in-house library (error tolerated: $0.3 \mathrm{Da}$ ) and the $\mathrm{MS}^{2}$ spectrum matches at least $85 \%$ with the reference spectrum (fragment ions and their relative intensities) of the in-house library.

\subsection{Validation of the quantification method}

The quantification method was validated based on the screening results of positive hits of suspected illegal antimicrobials. In total, 57 suspected illegal antimicrobials were screened by applying the validated screening method. The results confirmed that amoxicillin, cefalexin, penicillin $\mathrm{V}$, tetracycline, ampicillin, ciprofloxacin, ofloxacin, roxithromycin, clavulanic acid, benzathine penicillin G, ceftriaxone, doxycycline, griseofulvin, nitrofurantoin, norfloxacin, trimethoprim and sulfamethoxazole were indeed present in the respective illegal samples. Hence, the quantification method was validated for these 17 compounds (full validation for 12 and reduced validation for 5 compounds as explained in Section 2.5.2) and afterwards applied to quantify illegal antimicrobial samples. The method was validated for these 17 compounds at different wavelengths (See Table 1) selected based on the peak shapes and the absence of interference in the chromatogram.

\subsubsection{Selectivity}

To evaluate the method selectivity, the retention time and the UV spectrum of each compound were determined and monitored for 17 compounds. Constant retention times and selective UV spectra confirmed the method selectivity.

\subsubsection{Limits of detection and quantification}

LOD and the limit of quantification (LOQ) were determined according to the European Pharmacopoeia and the International Council on Harmonization (ICH) $[18,31]$. LOD and LOQ were obtained via experiments of serial dilutions. In general, signal-to-noise ratios of 3 and 10 are regarded as LOD and LOQ, respectively. The LOD and LOQ of these 17 compounds are given in Table 5. Ceftriaxone had the highest LOQ, $100 \mu \mathrm{g} / \mathrm{mL}$, which was sufficient for its quantification.

\subsubsection{Linearity of the calibration lines}

Ordinary least square linear regression was used to construct the calibration curves. The linearity was evaluated through $\mathrm{R}^{2}$ values, the quality coefficient (QC) and the p-values of the lack-of-fit (LOF) test. The latter is needed since $\mathrm{R}^{2}$ values and $\mathrm{QC}$ individually are not adequate enough to confirm the linearity. After being verified by the LOF test, the limits of $\mathrm{R}^{2} \geq 0.995$ and $\mathrm{QC} \leq 2.5 \%$ can be adopted for the system suitability test [32]. Within the selected calibration ranges, all 17 compounds had $\mathrm{R}^{2}$ values higher than 0.999 , QC values below $2.5 \%$ and p-values of the LOF test above 0.05 , as summarized in Table 5.

\subsubsection{Linearity, trueness, precision and accuracy}

Following ISO-17025, the developed method was validated by building accuracy profiles based upon the "total error" approach $[23,24]$ for the first set of positive illegal samples consisting of amoxicillin, cefalexin, penicillin V, tetracycline, ampicillin, ciprofloxacin, ofloxacin, roxithromycin, clavulanic acid, benzathine penicillin G, ceftriaxone and doxycycline. The accuracy profiles of these 12 compounds (shown in Fig. 4) are plotted to assess the present and future accuracy of the method. A reduced validation was performed for the other five antimicrobials as mentioned in Section 2.5.2.

3.4.4.1. Linearity of the results. It is compulsory that the theoretical concentration is linearly linked to the measured concentration $[27,33]$. With the investigation of the linear relationship for the 12 compounds individually, the results suggested that the relationship was linear since the $R^{2}$ values were above 0.9999 with an equation being close to $y=x$.

3.4.4.2. Trueness. In ISO guideline 5725, the trueness is defined as the closeness of agreement between the mean of a number of test results and the actual (true) value [34]. It is an estimate of the systematic error of the method and is indicated as relative bias. For the current method, the trueness is satisfactory since the maximum absolute value of the relative bias is $3.3 \%$ (See Table 6). All the relative biases for the 12 investigated compounds are located within the error limits of $[-5 \%$, $5 \%]$.

3.4.4.3. Precision. The precision of an analytical method is an estimate of the margin of random errors. It describes the closeness of agreement between a series of repeated measurements under the prescribed conditions [25]. The precision is expressed as the relative standard deviation (RSD) and may be considered at three levels: repeatability, intermediate precision and reproducibility. In this study, we investigated the intraday precision (repeatability) and the interday precision (intermediate precision). The repeatability of the method evaluates the precision under the same operating conditions in a short time interval, which was acquired from the RSD values of the triplicate measurements at each concentration level. The intermediate precision describes the within-laboratory variations, which is normally investigated on different days using different equipment and different analysts, though only the factor of the different days was taken into account here, due to practical reasons [26].

The results are shown in Table 6 . The RSD of repeatability and intermediate precision are below $1.3 \%$ and $1.7 \%$, respectively. In 

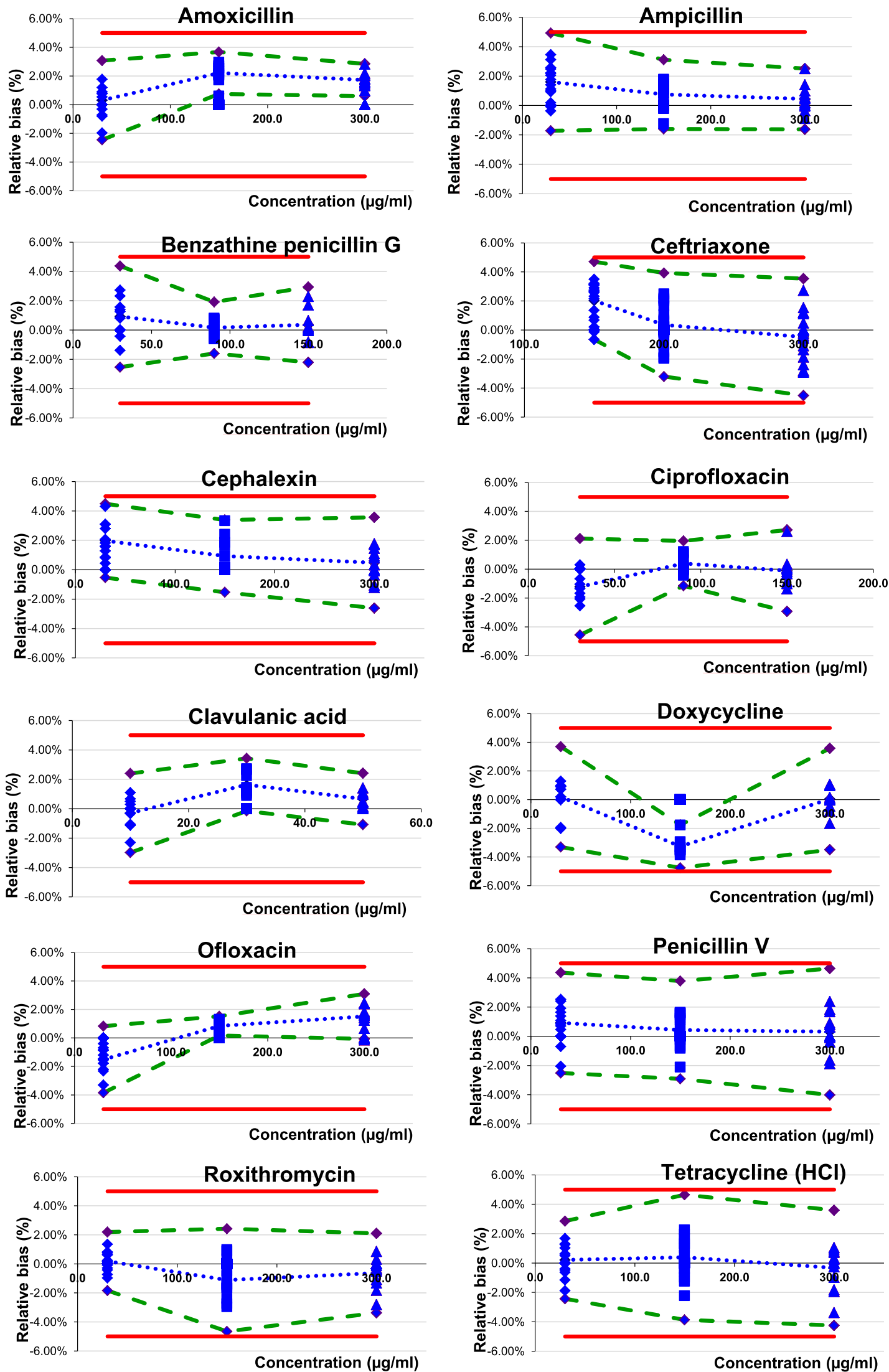

Fig. 4. Accuracy profiles of all 12 compounds with $95 \% \beta$. Relative bias (dashed blue line), $\beta$-expectation tolerance limits (dashed green line), $\pm 5 \%$ acceptance limits (solid red line) and relative back-calculated concentrations (dots). 
conclusion, the precision of the method is acceptable for all components.

3.4.4.4. Accuracy. To evaluate the accuracy of the analytical method, the $\beta$-expectation tolerance intervals were calculated based on the results of trueness and precision [24-26]. Accuracy takes the total error of the test results into consideration and predicts the future measurements. The acceptance limits of licensed pharmaceutical products are $[-5 \%, 5 \%]$, which is the same as the manufacturing limit [24]. The accuracy profiles for the 12 selected compounds are shown in Fig. 4. The obtained accuracy profiles indicate that $95 \%(\beta)$ of the sample measurements in the future will be located within the bias limit of $[-5 \%, 5 \%]$, which is acceptable for the intended use.

The measurement uncertainty describes the dispersion of the values that can be rationally ascribed to the analyte. The expanded uncertainty is determined with a $95 \%$ confidence level, representing an interval in which the unknown true value can be obtained. The relative expanded uncertainty as illustrated in Table 6 is calculated by dividing the expanded uncertainty by the corresponding concentration. The maximum value of relative expanded uncertainty was $3.7 \%$, which is acceptable and reasonable for the analysis of counterfeit antimicrobials.

\subsubsection{Reduced validation procedure}

Some illegal samples were received after the validation of the quantification method was finalized already. As no major problems were arisen for the first 12 compounds, it was decided to apply a reduced validation in terms of selectivity, LOD, LOQ, linearity and recovery for sulfamethoxazole, trimethoprim, norfloxacin, nitrofurantoin and griseofulvin. LOD, LOQ and linearity data are given in Table 5. The recovery test was performed on the simulated tablet for each antimicrobial separately. The preparation procedure has been explained in Section 2.3.2.2. All recoveries were $98 \%$ or higher (Table 3), indicating that the sample treatment procedure is also suitable for these antimicrobials.

\subsection{Market study}

In total, 57 illegal antimicrobials were collected. They contained 23 samples of amoxicillin, 9 samples of amoxicillin with clavulanic acid and 6 samples of azithromycin. Two samples were received for ampicillin, doxycycline, tetracycline and sulfamethoxazole with trimethoprim, respectively. For the rest of the 11 antimicrobials, 1 sample of each was collected.

\subsubsection{Identification of suspected illegal antimicrobials}

The validated screening method was applied to the 57 seized samples. The result indicated that these 57 samples were all positive for the claimed API.

\subsubsection{Quantification of the suspected illegal antimicrobials}

All suspected illegal antimicrobial samples that were positive for the indicated API could be successfully quantified using the validated UHPLC-DAD method, except for erythromycin and azithromycin. As mentioned in Section 3.1, these were quantified using the methods for related substances described in the European Pharmacopoeia [18]. The quantification results of the 57 samples are shown in Table 7, in which $49 \%$ of the samples contained amounts of API out of the limits of [95\%, 105\%]: $46 \%$ of the 57 samples were underdosed (ranging from $14.1 \%$ to $94.4 \%$ ), while $3 \%$ were overdosed. Nine out of the 23 samples of amoxicillin drug products had unqualified contents ranging from $82.5 \%$ to $94.0 \%$. Seven out of the 9 samples containing amoxicillin and clavulanic acid were severely underdosed for clavulanic acid. These findings concur previous ones indicating that samples containing this molecule were more prone to degradation [35]. This phenomenon was attributed to the chemical nature of clavulanic acid and could be the results of non-standard manufacturing, distribution or storage 
Table 7

An overview of the quantification results of 57 illegal samples.

\begin{tabular}{|c|c|c|c|c|}
\hline \multicolumn{2}{|l|}{ Identified compounds } & \multirow{2}{*}{$\begin{array}{l}\text { Amount } \\
\text { claimed } \\
(\mathrm{mg}) \\
250\end{array}$} & \multirow{2}{*}{$\begin{array}{l}\text { Amount } \\
\begin{array}{l}\text { present } \\
(\mathrm{mg})\end{array} \\
243\end{array}$} & \multirow{2}{*}{$\begin{array}{l}\text { Content (\%) } \\
97.1\end{array}$} \\
\hline Amoxicillin & Sample 1 & & & \\
\hline & Sample 2 & 250 & 234 & 93.6 \\
\hline & Sample 3 & 250 & 248 & 99.2 \\
\hline & Sample 4 & 250 & 242 & 96.6 \\
\hline & Sample 5 & 250 & 246 & 98.3 \\
\hline & Sample 6 & 250 & 235 & 94.0 \\
\hline & Sample 7 & 250 & 230 & 92.1 \\
\hline & Sample 8 & 500 & 482 & 96.3 \\
\hline & Sample 9 & 500 & 413 & 82.5 \\
\hline & Sample 10 & 500 & 491 & 98.3 \\
\hline & Sample 11 & 500 & 490 & 98.1 \\
\hline & Sample 12 & 500 & 442 & 88.4 \\
\hline & Sample 13 & 500 & 464 & 92.8 \\
\hline & Sample 14 & 500 & 493 & 98.6 \\
\hline & Sample 15 & 500 & 498 & 99.6 \\
\hline & Sample 16 & 500 & 479 & 95.8 \\
\hline & Sample 17 & 500 & 458 & 91.5 \\
\hline & Sample 18 & 500 & 485 & 97.0 \\
\hline & Sample 19 & 250 & 236 & 94.6 \\
\hline & Sample 20 & 7500 & 7000 & 93.7 \\
\hline & Sample 21 & 500 & 496 & 99.3 \\
\hline & Sample 22 & 500 & 480 & 95.9 \\
\hline & Sample 23 & 250 & 235 & 94.0 \\
\hline \multirow{9}{*}{$\begin{array}{l}\text { Amoxicillin/ } \\
\text { Clavulanic acid }\end{array}$} & Sample 1 & $875 / 125$ & $869 / 18$ & $99.3 / 14.1$ \\
\hline & Sample 2 & $875 / 125$ & $800 / 98$ & $91.4 / 78.5$ \\
\hline & Sample 3 & $875 / 125$ & $813 / 50$ & $92.9 / 40.0$ \\
\hline & Sample 4 & $875 / 125$ & $948 / 80$ & $108.3 / 63.7$ \\
\hline & Sample 5 & $500 / 125$ & $498 / 103$ & $99.5 / 82.1$ \\
\hline & Sample 6 & $500 / 125$ & $434 / 104$ & $86.8 / 83.4$ \\
\hline & Sample 7 & $500 / 125$ & $471 / 126$ & $94.2 / 100.6$ \\
\hline & Sample 8 & $500 / 125$ & $483 / 127$ & $96.6 / 101.8$ \\
\hline & Sample 9 & $250 / 125$ & $239 / 90$ & $95.6 / 72.3$ \\
\hline \multirow[t]{2}{*}{ Ampicillin } & Sample 1 & 250 & 224 & 89.8 \\
\hline & Sample 2 & 1000 & 784 & 78.4 \\
\hline $\begin{array}{l}\text { Benzathine Penicillin } \\
\text { G }\end{array}$ & Sample 1 & 1800 & 1795 & 99.7 \\
\hline Ceftriaxone & Sample 1 & 1000 & 1045 & 104.5 \\
\hline Cefalexin & Sample 1 & 500 & 486 & 97.3 \\
\hline Ciprofloxacin & Sample 1 & 500 & 512 & 102.4 \\
\hline \multirow[t]{2}{*}{ Doxycycline } & Sample 1 & 100 & 87 & 86.8 \\
\hline & Sample 2 & 100 & 98 & 98.3 \\
\hline Ofloxacin & Sample 1 & 200 & 189 & 94.4 \\
\hline Penicillin V & Sample 1 & 327 & 323 & 98.7 \\
\hline Roxithromycin & Sample 1 & 300 & 284 & 94.7 \\
\hline \multirow[t]{2}{*}{ Tetracycline $(\mathrm{HCl})$} & Sample 1 & 500 & 498 & 99.6 \\
\hline & Sample 2 & 250 & 244 & 97.4 \\
\hline \multirow{2}{*}{$\begin{array}{c}\text { Sulfamethoxazole/ } \\
\text { trimethoprim }\end{array}$} & Sample 1 & $800 / 160$ & $807 / 130$ & $100.8 / 81.2$ \\
\hline & Sample 2 & $800 / 160$ & $822 / 129$ & $102.7 / 80.6$ \\
\hline Nitrofurantoin & Sample 1 & 50 & 55 & 110.9 \\
\hline Norfloxacin & Sample 1 & 400 & 448 & 112.0 \\
\hline Griseofulvin & Sample 1 & 250 & 249 & 99.7 \\
\hline Erythromycin ${ }^{\mathrm{a}}$ & Sample 1 & 500 & 403 & 80.5 \\
\hline \multirow[t]{6}{*}{ Azithromycin ${ }^{\mathrm{a}}$} & Sample 1 & 250 & 260 & 104.0 \\
\hline & Sample 2 & 500 & 453 & 90.5 \\
\hline & Sample 3 & 500 & 513 & 102.6 \\
\hline & Sample 4 & 250 & 249 & 99.7 \\
\hline & Sample 5 & 500 & 511 & 102.2 \\
\hline & Sample 6 & 500 & 455 & 91.0 \\
\hline
\end{tabular}

${ }^{a}$ Analyzed by methods described in the European Pharmacopoeia.

procedures. Two samples of ampicillin, two samples of trimethoprim, one out of the two doxycycline samples, two out of 6 samples of azithromycin, one sample of erythromycin and one sample of ofloxacin were also found to contain insufficient API. An overview report indicated that $93 \%(14 / 15)$ of the studies concerning SF-antimicrobials addressed similar findings of insufficient amounts of API [36]. Underdosed antimicrobials show a lower efficacy and so may aggravate illness of patients and induce bacterial resistance [7]. In such a way, a potential threat may be posed to the public health.

\section{Conclusion}

A suitable LC-system for analysis of counterfeit antimicrobials was developed, optimized and validated for the identification via MS and quantification via UV/DAD. In this framework, a fast UHPLC-MS ${ }^{2}$ screening method taking $18 \mathrm{~min}$ was developed for the identification of 35 antimicrobials and 1 beta-lactamase inhibitor. This method has been validated in terms of selectivity, sensitivity and matrix effects. In addition, a UHPLC-DAD quantification method with an analysis time of $32 \mathrm{~min}$ was developed for 31 antimicrobials and 1 beta-lactamase inhibitor. Neomycin, gentamicin, erythromycin and azithromycin were excluded from the quantification method due to their insufficient UV absorbance. The quantification method was validated for samples yielding positive hits in the screening test. As a result, it was validated for 16 antimicrobials and 1 beta-lactamase inhibitor through a full or reduced validation process.

The validated screening method and quantification method have been successfully applied to 57 suspicious illegal antimicrobials seized by FAMHP. All suspected samples contained the indicated API. Whereas about half of the suspected illegal antimicrobials displayed sufficient amounts of API, some (3\%) were overdosed, while others (46\%) expressed insufficient amounts of API. It was noticed that suspected samples of amoxicillin with clavulanic acid had a quality issue since most of them were underdosed for the beta-lactamase inhibitor.

Taken together, the two described methods demonstrate that they are suitable for quality control of both legal and illegal antimicrobials. They are fast, not labor intensive and make it possible to analyze a wide range of antimicrobials simultaneously, enabling a more in-dept analysis of these SF-antimicrobials.

\section{Acknowledgements}

The authors would like to thank the China Scholarship Council, China (CSC, File No. 201607650019). We also would like to thank Angélique Kamugisha and Willy Van De Wauw for the technical support, and Steven Janvier for the advice on the research and the article.

\section{Conflicts of interests}

The authors state that there is no conflict of interest.

\section{References}

[1] World Health Organization, A study on the public heath and socioeconomic impact of substandard and falsified medical products, 2017. 〈http://www.who.int/ medicines/regulation/ssffc/publications/se-study-sf/en/〉 (Accessed 6 June 2018)

[2] World Health Organization, WHO global surveillance and monitoring system for substandard and falsified medical products, Geneva. 〈http://www.who.int/ medicines/regulation/ssffc/publications/GSMS_Report.pdf?Ua $=1>$ (Accessed 13 July 2018).

[3] A. Delepierre, A. Gayot, A. Carpentier, Update on counterfeit antibiotics worldwide; Public health risks, Med. Mal. Infect. 42 (2012) 247-255, https://doi.org/10.1016/ j.medmal.2012.04.007.

[4] Grand view research, Antibiotics market size to reach $\$ 57.0$ billion by 2024,2016 . 〈https://www.grandviewresearch.com/press-release/global-antibiotic-market〉 (Accessed 17 July 2018).

[5] B.J. Venhuis, P.H.J. Keizers, R. Klausmann, I. Hegger, Operation resistance: a snapshot of falsified antibiotics and biopharmaceutical injectables in Europe, Drug Test. Anal. 8 (2016) 398-401, https://doi.org/10.1002/dta.1888.

[6] WHO, Substandard and falsified medical products. 〈http://www.who.int/ mediacentre/factsheets/fs275/en/ > (Accessed 20 July 2018).

[7] T. Kelesidis, M.E. Falagas, Substandard/counterfeit antimicrobial drugs, Clin. Microbiol. Rev. 28 (2015) 443-464, https://doi.org/10.1128/CMR.00072-14.

[8] World Health Organization, WHO global strategy for containment of antimicrobial resistance, Geneva, World Health Organization, 2001. 〈http://www.who.int/iris/ handle/10665/66872> (Accessed 1 August 2018).

[9] E. Patyra, C. Nebot, R.E. Gavilán, A. Cepeda, K. Kwiatek, Development and validation of multi-residue and multi-class method for antibacterial substances analysis in non-target feed by liquid chromatography-tandem mass spectrometry, Food Addit. Contam. - Part A Chem. Anal. Control. Expo. Risk Assess. 35 (2018) 467-478, https://doi.org/10.1080/19440049.2017.1414961.

[10] A. Chahbouni, F.A.M. Van den Dungen, R.M. Vos, J.C.G. Den Burger, A. Sinjewel, 
A.J. Wilhelm, A.I. Veldkamp, E.L. Swart, M.M. Van Weissenbruch, An UPLC-MS detection method for the quantification of five antibiotics in human plasma, Bioanalysis 7 (2015) 2321-2329, https://doi.org/10.4155/bio.15.121.

[11] M. Gros, S. Rodríguez-Mozaz, D. Barceló, Rapid analysis of multiclass antibiotic residues and some of their metabolites in hospital, urban wastewater and river water by ultra-high-performance liquid chromatography coupled to quadrupolelinear ion trap tandem mass spectrometry, J. Chromatogr. A 1292 (2013) 173-188, https://doi.org/10.1016/j.chroma.2012.12.072.

[12] L. Tong, H. Liu, C. Xie, M. Li, Quantitative analysis of antibiotics in aquifer sediments by liquid chromatography coupled to high resolution mass spectrometry, J. Chromatogr. A 1452 (2016) 58-66, https://doi.org/10.1016/j.chroma.2016.05. 027.

[13] T. Van den Meersche, E. Van Pamel, C. Van Poucke, L. Herman, M. Heyndrickx, G. Rasschaert, E. Daeseleire, Development, validation and application of an ultra high performance liquid chromatographic-tandem mass spectrometric method for the simultaneous detection and quantification of five different classes of veterinary antibiotics in swine manure, J. Chromatogr. A 1429 (2016) 248-257, https://doi. org/10.1016/j.chroma.2015.12.046.

[14] İ. Kivrak, Ş. Kivrak, M. Harmandar, Development of a rapid method for the determination of antibiotic residues in honey using UPLC-ESI-MS/MS, Food Sci. Technol. 36 (2016) 90-96, https://doi.org/10.1590/1678-457X.0037.

[15] L. Chiesa, S. Panseri, E. Pasquale, R. Malandra, R. Pavlovic, F. Arioli, Validated multiclass targeted determination of antibiotics in fish with high performance liquid chromatography-bench top quadrupole orbitrap hybrid mass spectrometry, Food Chem. 258 (2018) 222-230, https://doi.org/10.1016/j.foodchem.2018.03. 072.

[16] D. Shin, H.S. Kang, J. Jeong, J. Kim, W.J. Choe, K.S. Lee, G.S. Rhee, Multi-residue determination of veterinary drugs in fishery products using liquid chromatographytandem mass spectrometry, Food Anal. Methods 11 (2018) 1815-1831, https://doi. org/10.1007/s12161-018-1179-0.

[17] E. Dubreil, S. Gautier, M.P. Fourmond, M. Bessiral, M. Gaugain, E. Verdon, D. Pessel, Validation approach for a fast and simple targeted screening method for 75 antibiotics in meat and aquaculture products using LC-MS/MS, Food Addit. Contam. - Part A Chem. Anal. Control Expo. Risk Assess. 34 (2017) 453-468, https://doi.org/10.1080/19440049.2016.1230278.

[18] European Pharmacopoeia, 9th ed., Council of Europe, Strasbourg, France, 2018.

[19] Y. Chen, C. Hu, J. Qu, M. Yang, Photodegradation of tetracycline and formation of reactive oxygen species in aqueous tetracycline solution under simulated sunlight irradiation, J. Photochem. Photobiol. A Chem. 197 (2008) 81-87, https://doi.org/ 10.1016/j.jphotochem.2007.12.007.

[20] C. Vanhee, E. Tuenter, A. Kamugisha, M. Canfyn, G. Moens, P. Courselle, L. Pieters, E. Deconinck, V. Exarchou, Identification and quantification methodology for the analysis of suspected illegal dietary supplements: reference standard or no reference standard, that's the question, J. Forensic Toxicol. Pharmacol. 07 (2018) 1-7, https://doi.org/10.4172/2325-9841.1000156.

[21] European Commission, Method validation and quality control procedures for pesticide residues analysis in food and feed, document No. SANCO/12495/2011, Brussels, 2012. 〈http://www.crl-pesticides.eu/library/docs/fv/SANCO124952011.pdf $>$ (Accessed 8 August 2018).

[22] CRLs, Guidelines for the validation of screening methods for residues of veterinary medicines, Community reference laboratories residues, 2010. 〈https://ec.europa. $\mathrm{eu} / \mathrm{food} / \mathrm{sites} / \mathrm{food} /$ files/safety/docs/cs vet-med-residues guideline validation screening_en.pdf> (Accessed 8 August 2018).
[23] ISO/IEC, 17025. General requirements for the competence of testing and calibration laboratories, ISO, Geneva, 2017. 〈www.iso.org

[24] M. Feinberg, Validation of analytical methods based on accuracy profiles, J. Chromatogr. A 1158 (2007) 174-183, https://doi.org/10.1016/j.chroma.2007.02. 021.

[25] P. Hubert, J.J. Nguyen-Huu, B. Boulanger, E. Chapuzet, P. Chiap, N. Cohen, P.A. Compagnon, W. Dewé, M. Feinberg, M. Lallier, M. Laurentie, N. Mercier, G. Muzard, C. Nivet, L. Valat, Harmonization of strategies for the validation of quantitative analytical procedures: a SFSTP proposal - Part I, J. Pharm. Biomed. Anal. 36 (2007) 579-586, https://doi.org/10.1016/j.jpba.2004.07.027.

[26] A. Gustavo González, M. Ángeles Herrador, A practical guide to analytical method validation, including measurement uncertainty and accuracy profiles, TrAC - Trends Anal. Chem. 26 (2007) 227-238, https://doi.org/10.1016/j.trac.2007.01.009.

[27] P.Y. Sacré, E. Deconinck, P. Chiap, J. Crommen, F. Mansion, E. Rozet, P. Courselle, J.O. De Beer, Development and validation of a ultra-high-performance liquid chromatography-UV method for the detection and quantification of erectile dysfunction drugs and some of their analogues found in counterfeit medicines, J. Chromatogr. A 1218 (2011) 6439-6447, https://doi.org/10.1016/j.chroma.2011. 07.029.

[28] M. Ibáñez, C. Guerrero, J.V. Sancho, F. Hernández, Screening of antibiotics in surface and wastewater samples by ultra-high-pressure liquid chromatography coupled to hybrid quadrupole time-of-flight mass spectrometry, J. Chromatogr. A 1216 (2009) 2529-2539, https://doi.org/10.1016/j.chroma.2009.01.073.

[29] A. Junza, N. Dorival-García, A. Zafra-Gómez, D. Barrón, O. Ballesteros, J. Barbosa, A. Navalón, Multiclass method for the determination of quinolones and $\beta$-lactams, in raw cow milk using dispersive liquid-liquid microextraction and ultra high performance liquid chromatography-tandem mass spectrometry, J. Chromatogr. A 1356 (2014) 10-22, https://doi.org/10.1016/j.chroma.2014.06.034.

[30] X.L. Hou, Y.L. Wu, Y. Lv, X.Q. Xu, J. Zhao, T. Yang, Development and validation of an ultra high performance liquid chromatography tandem mass spectrometry method for determination of 10 cephalosporins and desacetylcefapirin in milk, J. Chromatogr. B Anal. Technol. Biomed. Life Sci. 931 (2013) 6-11, https://doi.org/ 10.1016/j.jchromb.2013.05.006.

[31] ICH, Q2(r1), International Council on Harmonization: Validation of Analytical Procedures: Text and Methodology, 1996.

[32] J. Van Loco, M. Elskens, C. Croux, H. Beernaert, Linearity of calibration curves: use and misuse of the correlation coefficient, Accredit. Qual. Assur. 7 (2002) 281-285, https://doi.org/10.1007/s00769-002-0487-6.

[33] B. Desmedt, V. Rogiers, P. Courselle, J.O. De Beer, K. De Paepe, E. Deconinck, Development and validation of a fast chromatographic method for screening and quantification of legal and illegal skin whitening agents, J. Pharm. Biomed. Anal. 83 (2013) 82-88, https://doi.org/10.1016/j.jpba.2013.04.020.

[34] ISO Guideline 5725, Part 1: General principles and definitions, in: Accuracy (trueness and precision) of Measurement Methods and Results, 1994. 〈www.iso. org $>$

[35] M.H. Khan, K. Hatanaka, T. Sovannarith, N. Nivanna, L.C.C. Casas, N. Yoshida, H. Tsuboi, T. Tanimoto, K. Kimura, Effects of packaging and storage conditions on the quality of amoxicillin-clavulanic acid-an analysis of Cambodian samples, BMC Pharmacol. Toxicol. 14 (2013), https://doi.org/10.1186/2050-6511-14-33.

[36] T. Almuzaini, I. Choonara, H. Sammons, Substandard and counterfeit medicines: a systematic review of the literature, BMJ Open (2013) 1-7, https://doi.org/10. 1136/bmjopen-2013-002923. 